\title{
Multiple weighted estimates for vector-valued commutators of multilinear square functions
}

\section{Zengyan Si}

School of Mathematics and Information Science, Henan Polytechnic University, Jiaozuo 454000, People's Republic of China.

Communicated by Y. Hu

\begin{abstract}
Let $T$ be the multilinear square function with a kernel of Dini's type and $T_{q}$ be the vector-valued version of $T$. In this paper, we obtain the weighted strong type and weighted end-point weak type estimates for the commutators of $T_{q}$ respectively if the kernels satisfies L $\log \mathrm{L}^{\mathrm{l}}$-Dini type conditions. (C)2017 All rights reserved.
\end{abstract}

Keywords: Multilinear square functions, vector-valued inequality, weights.

2010 MSC: 42B25, 47G10.

\section{Introduction}

Let $K_{t}\left(x, y_{1}, \ldots, y_{m}\right)$ be a locally integrable function defined away from the diagonal $x=y_{1}=\cdots=y_{m}$ in $\left(\mathbb{R}^{n}\right)^{m+1}$. For any $x \notin \bigcap_{j=1}^{m} \operatorname{supp}_{j}$, and each $f_{j} \in C_{c}^{\infty}\left(\mathbb{R}^{n}\right)$, the multilinear square function is given by

$$
T(\vec{f})(x)=\left(\int_{0}^{\infty}\left|\int_{\left(\mathbb{R}^{n}\right)^{m}} K_{t}\left(x, y_{1}, \ldots, y_{m}\right) \prod_{j=1}^{m} f_{j}\left(y_{j}\right) d y_{1} \ldots d y_{m}\right|^{2} \frac{d t}{t}\right)^{\frac{1}{2}} .
$$

This kind of operator has important applications in PDEs and other fields, we refer to $[2,4-7,9,12,13]$ and the references therein. For instance, Fabes et al. [5] obtained a collection of multilinear LittlewoodPaley estimates, which they then applied to two problems in partial differential equations. The first problem is the estimation of the square root of an elliptic operator in divergence form, and the second is the estimation of solutions to the Cauchy problem for nondivergence form parabolic equations.

In order to state our main results, let us recall some notations and definitions.

Suppose that $\bar{\omega}(t):[0, \infty) \mapsto[0, \infty)$ is a nondecreasing function with $0<\bar{\omega}(1)<\infty$. For $a>0$, we say that $\bar{\omega} \in \operatorname{Dini}(a)$, if

$$
|\bar{\omega}|_{\operatorname{Dini}(a)}=\int_{0}^{1} \bar{\omega}^{a}(t) \frac{d t}{t}<\infty .
$$

Email address: zengyan@hpu.edu.cn (Zengyan Si)

doi:10.22436/jnsa.010.06.20 
Suppose that there is a positive constant $A$ such that the kernel $K_{t}$ satisfies the following conditions. Size condition:

$$
\left(\int_{0}^{\infty}\left|K_{t}\left(x, y_{1}, \cdots, y_{m}\right)\right|^{2} \frac{d t}{t}\right)^{\frac{1}{2}} \leqslant \frac{A}{\left(\sum_{j=1}^{m}\left|x-y_{j}\right|\right)^{m n}}
$$

Smoothness condition:

$$
\left(\int_{0}^{\infty}\left|K_{t}\left(z, y_{1}, \cdots, y_{m}\right)-K_{t}\left(x, y_{1}, \cdots, y_{m}\right)\right|^{2} \frac{d t}{t}\right)^{\frac{1}{2}} \leqslant \frac{A}{\left(\sum_{j=1}^{m}\left|x-y_{j}\right|\right)^{m n}} \bar{\omega}\left(\frac{|z-x|}{\sum_{j=1}^{m}\left|x-y_{j}\right|}\right),
$$

whenever $|z-x| \leqslant \frac{1}{2} \max _{j=1}^{m}\left|x-y_{j}\right|$, and

$$
\begin{aligned}
\left(\int_{0}^{\infty} \mid K_{t}\left(x, y_{1}, \ldots, y_{j}, \ldots, y_{m}\right)\right. & \left.-\left.K_{t}\left(x, y_{1}, \ldots, y_{j}^{\prime}, \ldots, y_{m}\right)\right|^{2} \frac{d t}{t}\right)^{\frac{1}{2}} \\
& \leqslant \frac{A}{\left(\sum_{j=1}^{m}\left|x-y_{j}\right|\right)^{m n}} \bar{\omega}\left(\frac{\left|y_{j}-y_{j}^{\prime}\right|}{\sum_{j=1}^{m}\left|x-y_{j}\right|}\right),
\end{aligned}
$$

whenever $\left|y_{j}-y_{j}^{\prime}\right| \leqslant \frac{1}{2} \max _{j=1}^{m}\left|x-y_{j}\right|$.

In this paper, we always assume that $T$ can be extended to bounded operators from $L^{q_{1}} \times \cdots \times L^{q_{m}}$ to L $^{\mathrm{q}}$ for some $1<\mathrm{q}, \mathrm{q}_{1}, \cdots, \mathrm{q}_{\mathrm{m}}<\infty$ with $\frac{1}{\mathrm{q}_{1}}+\cdots+\frac{1}{\mathrm{q}_{\mathrm{m}}}=\frac{1}{\mathrm{q}}$.

Remark 1.1. When $\bar{\omega}(x)=x^{\gamma}$ for some $\gamma>0$, the boundedness of multilinear square function were studied by Xue et al. [12].

Remark 1.2. Let $T$ be a multilinear square function with a kernel satisfying Dini(1) condition. Si et al. [11] showed that $T$ is bounded from $L^{1} \times \cdots \times L^{1}$ to $L^{\frac{1}{m}}, \infty$. Secondly, they obtained that, if each $p_{i}>1$, then $T$ is bounded from $L^{p_{1}}\left(\omega_{1}\right) \times \cdots \times L^{p_{m}}\left(\omega_{m}\right)$ to $L^{p}\left(v_{\vec{\omega}}\right)$ and if there is a $p_{i}=1$, then $T$ is bounded from $L^{p_{1}}\left(\omega_{1}\right) \times \cdots \times L^{p_{m}}\left(\omega_{m}\right)$ to $L^{p, \infty}\left(v_{\vec{\omega}}\right)$, where $v_{\vec{\omega}}=\prod_{i=1}^{m} \omega_{i}^{p / p_{i}}$ (see Section 2 Definition 2.1 for the definition).

The main purpose of this paper is to study the boundedness properties of vector-valued commutator $\mathrm{T}_{\Pi \vec{b}, q}$ generated by $\mathrm{T}$ and BMO functions. Let $\vec{b}=\left(b_{1}, \ldots, b_{l}\right) \in(B M O)^{l}$. For any given positive integer $l$ with $1 \leqslant l \leqslant m$, the commutators associated with $T$ are defined by

$$
T_{\Pi \vec{b}}(\vec{f})(x)=\left(\int_{0}^{\infty}\left|\int_{\left(\mathbb{R}^{n}\right)^{m}} \prod_{j=1}^{l}\left[b_{j}(x)-b_{j}\left(y_{j}\right)\right] K_{t}\left(x, y_{1}, \ldots, y_{m}\right) \prod_{j=1}^{m} f_{j}\left(y_{j}\right) d y_{1} \ldots d y_{m}\right|^{2} \frac{d t}{t}\right)^{1 / 2} .
$$

The vector-valued version of the commutator $T_{\Pi \vec{b}}$ can be defined by

$$
\mathrm{T}_{\Pi \overrightarrow{\mathrm{b}}, \mathrm{q}}(\overrightarrow{\mathrm{f}})(\mathrm{x})=\left|\mathrm{T}_{\Pi \overrightarrow{\mathrm{b}}}(\vec{f})(\mathrm{x})\right|_{\mathrm{q}}=\left(\sum_{\mathrm{k}=1}^{\infty}\left|\mathrm{T}_{\Pi \overrightarrow{\mathrm{b}}}\left(\mathrm{f}_{1 \mathrm{k}}, \cdots, \mathrm{f}_{\mathrm{mk}}\right)(\mathrm{x})\right|^{\mathrm{q}}\right)^{1 / \mathrm{q}},
$$

where $\vec{f}=\left(f_{1}, \ldots, f_{m}\right)$ with $f_{i}=\left\{f_{i k}\right\}_{k=1}^{\infty}$ for $i=1, \cdots, m$.

We get the following strong and end-point estimates for $T_{\Pi \vec{b}, q}$.

Theorem 1.3. Let $1 / \mathrm{m}<\mathrm{p}<\infty, \frac{1}{\mathrm{p}}=\frac{1}{\mathrm{p}_{1}}+\cdots+\frac{1}{\mathrm{p}_{\mathrm{m}}}$ with $1<\mathrm{p}_{1}, \cdots, \mathrm{p}_{\mathrm{m}}<\infty, 1 / \mathrm{m}<\mathrm{q}<\infty$ and $\frac{1}{q_{1}}+\cdots+\frac{1}{q_{m}}=\frac{1}{q}$ with $1<q_{1}, \cdots, q_{m}<\infty$. If $\vec{\omega} \in A_{\vec{p}}, \vec{b} \in(B M O)^{l}$ and $\bar{\omega}$ satisfies

$$
|\bar{\omega}|_{\mathrm{L} \log \mathrm{L}^{\mathrm{l}}-\mathrm{Dini}}=\int_{0}^{1} \bar{\omega}(\mathrm{t})\left(1+\log \frac{1}{\mathrm{t}}\right) \frac{\mathrm{dt}}{\mathrm{t}}<\infty,
$$

then

$$
\left\|T_{\Pi \vec{b}, q}(\vec{f})\right\|_{L^{p}\left(v_{\vec{w}}\right)} \leqslant C \prod_{j=1}^{l}\left\|b_{j}\right\|_{B M O} \prod_{j=1}^{m}\left\|\left|f_{j}\right|_{q_{j}}\right\|_{L^{p_{j}\left(w_{j}\right)}} .
$$


Theorem 1.4. Let $1 / \mathrm{m}<\mathrm{q}<\infty$ and $\frac{1}{\mathrm{q}_{1}}+\cdots+\frac{1}{\mathrm{q}_{\mathrm{m}}}=\frac{1}{\mathrm{q}}$ with $1<\mathrm{q}_{1}, \cdots, \mathrm{q}_{\mathrm{m}}<\infty$. If $\vec{\omega} \in \mathrm{A}_{(1, \cdots, 1)}$, $\overrightarrow{\mathrm{b}} \in(\mathrm{BMO})^{\mathrm{l}}$ and $\overline{\mathrm{\omega}}$ satisfies the condition (1.2), then

$$
v_{\vec{w}}\left(\left\{x \in \mathbb{R}^{n}: T_{\Pi \vec{b}, q}(\vec{f})(x)>t^{m}\right\}\right) \leqslant C\left(\prod_{j=1}^{m} \int_{\mathbb{R}^{n}} \Phi^{(m)}\left(\frac{\left|f_{j}\right|_{q_{j}}\left(y_{j}\right)}{t}\right) \omega_{j}\left(y_{j}\right) d y_{j}\right)^{1 / m},
$$

where $\Phi^{(m)}=\overbrace{\Phi \circ \cdots \circ \Phi}^{m}$ and $\Phi(t)=t\left(1+\log ^{+} t\right)$.

Throughout this paper, $\mathrm{C}$ denotes a positive constant that is independent of the main parameters involved but whose value may differ from line to line.

\section{Proofs of Theorem 1.3 and Theorem 1.4}

Let us begin with the definition of multiple-weights introduced by Lerner et al. [8].

Definition 2.1 ([8]). Let $1 \leqslant p_{1}, \cdots, p_{m}<\infty$, and $1 / p=1 / p_{1}+\cdots+1 / p_{m}$. Given $\vec{\omega}=\left(\omega_{1}, \cdots, \omega_{m}\right)$, set $v_{\vec{\omega}}=\prod_{i=1}^{m} \omega_{i}^{p / p_{i}}$. We say that $\vec{\omega}$ satisfies the $A_{\vec{p}}$ condition if

$$
\sup _{\mathrm{Q}}\left(\frac{1}{|\mathrm{Q}|} \int_{\mathrm{Q}} \prod_{i=1}^{\mathrm{m}} \omega_{i}^{\frac{\mathrm{p}}{\mathrm{p}_{i}}}\right)^{\frac{1}{\mathrm{p}}} \prod_{i=1}^{\mathrm{m}}\left(\frac{1}{|\mathrm{Q}|} \int_{\mathrm{Q}} \omega_{i}^{1-\mathrm{p}_{i}^{\prime}}\right)^{\frac{1}{p_{i}^{\prime}}}<\infty,
$$

when $p_{i}=1,\left(\frac{1}{|Q|} \int_{Q} \omega_{i}^{1-p_{i}^{\prime}}\right)^{\frac{1}{p_{i}^{\prime}}}$ is understood as $\left(\inf _{Q} \omega_{i}\right)^{-1}$.

Definition 2.2. For $1 \leqslant l \leqslant m$, the maximal operators $\mathcal{M}_{\mathrm{L}(\log \mathrm{L})}^{\mathrm{l}}\left(|\overrightarrow{\mathrm{f}}|_{\mathrm{q}}\right)$ and $\mathcal{M}\left(|\vec{f}|_{\mathrm{q}}\right)$ are respectively defined by

$$
\mathcal{M}_{\mathrm{L}(\log \mathrm{L})}^{\mathrm{l}}\left(|\overrightarrow{\mathrm{f}}|_{\mathrm{q}}\right)(\mathrm{x})=\sup _{\mathrm{Q} \ni \mathrm{x}} \prod_{j=1}^{\mathrm{l}}||\left|\mathrm{f}_{\mathbf{j}}\right|_{\mathbf{q}_{j}} \|_{\mathrm{L}(\log \mathrm{L}), \mathrm{Q}} \prod_{j=l+1}^{\mathrm{m}} \frac{1}{|\mathrm{Q}|} \int_{\mathrm{Q}}\left|\mathrm{f}_{\mathbf{j}}\right|_{\mathbf{q}_{j}}
$$

and

$$
\mathcal{M}\left(|\vec{f}|_{q}\right)(x)=\sup _{Q \ni x} \prod_{j=1}^{m} \frac{1}{|Q|} \int_{Q}\left|f_{j}\right|_{q_{j}},
$$

where the supremum is taken over all the cubes containing $x$.

We can control multilinear square function by using maximal operators in the following way.

Lemma 2.3 ([11]). Let $\bar{\omega} \in \operatorname{Dini}(1)$ and $0<\delta<\frac{1}{m}$. For any compact supported $f_{j}, j=1, \ldots, m$, we have

$$
M_{\delta}^{\sharp} T(\vec{f})(x) \leqslant \operatorname{C\mathcal {M}}(\vec{f})(x) .
$$

The above lemma can be used to establish the vector-valued inequalities for multilinear square function. The vector-valued multilinear square function $T_{q}$ associated is defined by

$$
\mathrm{T}_{\mathrm{q}}(\vec{f})(x)=|\mathrm{T}(\vec{f})(x)|_{\mathrm{q}}=\left(\sum_{k=1}^{\infty}\left|\mathrm{T}\left(f_{1 k}, \cdots, f_{m k}\right)(x)\right|^{q}\right)^{1 / q},
$$

where $\vec{f}=\left(f_{1}, \ldots, f_{m}\right)$ with $f_{i}=\left\{f_{i k}\right\}_{k=1}^{\infty}$ for $i=1, \cdots, m$.

Theorem 2.4. Let $1 \leqslant \mathrm{p}_{1}, \cdots, \mathrm{p}_{\mathrm{m}}<\infty, 1<\mathrm{q}_{1}, \cdots, \mathrm{q}_{\mathrm{m}}<\infty$ and $0<\mathrm{p}, \mathrm{q}<\infty$ such that $\frac{1}{\mathrm{p}}=\frac{1}{\mathrm{p}_{1}}+\cdots+$ $\frac{1}{\mathrm{p}_{\mathrm{m}}}, \frac{1}{\mathrm{q}}=\frac{1}{\mathrm{q}_{1}}+\cdots+\frac{1}{\mathrm{q}_{\mathrm{m}}}$. Suppose that $\bar{\omega} \in \operatorname{Dini}(1)$, then we have 
(i) If $1<p_{1}, \ldots, p_{m}<\infty$ and $\omega \in A_{p_{1}} \cap \cdots \cap A_{p_{m}}$, then

$$
\left\|T_{q}(\vec{f})\right\|_{L^{p}(\omega)} \leqslant C \prod_{j=1}^{m}\left\|\left.f_{j}\right|_{q_{j}}\right\|_{L^{p_{j}(\omega)}} .
$$

(ii) If at least one $p_{j}=1$ and $\omega \in A_{1}$, then

$$
\left\|T_{q}(\vec{f})\right\|_{L^{p, \infty}(\omega)} \leqslant C \prod_{j=1}^{m}\left|\left\|\left.f_{j}\right|_{q_{j}}\right\|_{L^{p_{j}}(\omega)} .\right.
$$

Proof. By using Lemma 2.3 and a standard argument, we can deduce that

$$
\|T \vec{f}\|_{L^{p}(\omega)} \leqslant C\left\|\prod_{j=1}^{m} M\left(f_{j}\right)\right\|_{L^{p}(\omega)}
$$

We apply the extrapolation theorems $\left(\left[3\right.\right.$, Theorem 2.1]) to $\left(T \vec{f}, \prod_{j=1}^{m} M f_{j}\right) \in \mathcal{F}$, then by the vector-valued inequality for the Hardy-Littlewood maximal operator $M$ ([1, Theorem 3.1]) we get the conclusion of Theorem 2.4.

To prove Theorems 1.3 and 1.4, we need the following preliminary lemmas.

Lemma 2.5. Let $0<\delta<1 / \mathrm{m}, 1 / \mathrm{m}<\mathrm{q}<\infty$ and $1 / \mathrm{q}=1 / \mathrm{q}_{1}+\cdots+1 / \mathrm{q}_{\mathrm{m}}$ with $1<\mathrm{q}_{1}, \cdots, \mathrm{q}_{\mathrm{m}}<\infty$. If $\bar{\omega}$ satisfies the condition (1.2), then

$$
M_{\delta}^{\sharp}\left(T_{q}(\vec{f})\right)(x) \leqslant \operatorname{C\mathcal {M}}\left(|\vec{f}|_{q}\right)(x)
$$

holds for any smooth vector function $\left\{\overrightarrow{\mathrm{f}}_{\mathrm{k}}\right\}_{\mathrm{k}=1}^{\infty}$ and any $\mathrm{x} \in \mathbb{R}^{\mathrm{n}}$.

Proof. For any fixed $x \in \mathbb{R}^{n}$, let $\mathrm{Q}$ be a cube which contains $x$ with side length $l(\mathrm{Q})$. We decompose $\vec{f}=\vec{f}^{0}+\sum_{\vec{\alpha}, \vec{\alpha} \neq \overrightarrow{0}} \overrightarrow{f^{\alpha}}$, where $\vec{f}^{0}=\vec{f} \chi_{8 \sqrt{n} Q}=\left(f_{1} \chi_{8 \sqrt{n}}, \cdots, f_{m} \chi_{8 \sqrt{n} Q}\right)$ and $\vec{\alpha}=\left(\alpha_{1}, \ldots, \alpha_{m}\right), \alpha_{i}=0$ or $\infty$. Set $C=|c|_{q}=\left(\sum_{k \geqslant 1}\left|c_{k}\right|^{q}\right)^{1 / q}$. Then we have

$$
\begin{aligned}
\left(\frac{1}{|\mathrm{Q}|} \int_{\mathrm{Q}}\left|\mathrm{T}_{\mathrm{q}}(\overrightarrow{\mathrm{f}})(\mathrm{y})\right|^{\delta}-|\mathrm{C}|^{\delta} \mid \mathrm{dy}\right)^{\frac{1}{\delta}} & \leqslant \mathrm{C}\left(\frac{1}{|\mathrm{Q}|} \int_{\mathrm{Q}}\left|\mathrm{T}_{\mathrm{q}}\left(\overrightarrow{\mathrm{f}^{0}}\right)(\mathrm{y})\right|^{\delta} \mathrm{dy}\right)^{\frac{1}{\delta}}+\left(\frac{1}{|\mathrm{Q}|} \int_{\mathrm{Q}}\left|\sum_{\vec{\alpha}, \vec{\alpha} \neq \overrightarrow{0}} \mathrm{~T}\left(\overrightarrow{\mathrm{f}^{\alpha}}\right)(\mathrm{y})-\mathrm{c}\right|_{\mathrm{q}}^{\delta} \mathrm{dy}\right)^{\frac{1}{\delta}} \\
& =\mathrm{U}_{1}+\mathrm{U}_{2} .
\end{aligned}
$$

For $\mathrm{U}_{1}$, we apply Kolmogorov's inequality and Theorem 2.4 to get

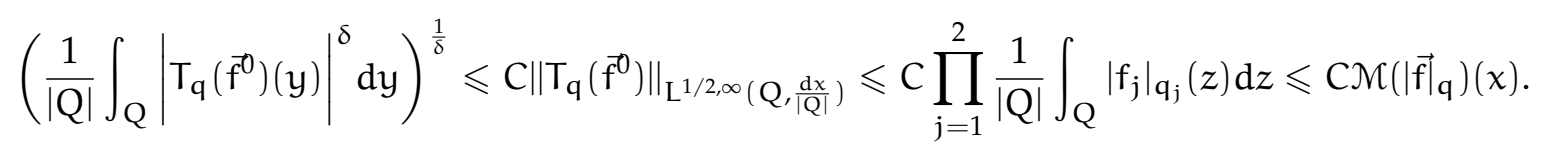

For $U_{2}$, we choose $c=\sum_{\vec{\alpha}, \vec{\alpha} \neq \overrightarrow{0}} c^{\vec{\alpha}}$, where $c^{\vec{\alpha}}=T\left(f_{1 k}^{\alpha_{1}}, \cdots, f_{m k}^{\alpha_{m}}\right)(x)$. It is obvious that $U_{2} \leqslant \sum_{\vec{\alpha}, \vec{\alpha} \neq \overrightarrow{0}} U_{2 \vec{\alpha}}$, where

$$
\mathrm{U}_{2 \vec{\alpha}}=\left(\frac{1}{|\mathrm{Q}|} \int_{\mathrm{Q}}\left|\mathrm{T}\left(\mathrm{f}_{1 \mathrm{k}}^{\alpha_{1}}, \ldots, \mathrm{f}_{\mathrm{mk}}^{\alpha_{\mathrm{m}}}\right)(\mathrm{y})-\mathrm{T}\left(\mathrm{f}_{1 \mathrm{k}}^{\alpha_{1}}, \ldots, f_{\mathrm{mk}}^{\alpha_{\mathrm{m}}}\right)(x)\right|_{\mathrm{q}}^{\delta} \mathrm{dy}\right)^{\frac{1}{\delta}}
$$

Next, we estimate the typical term $\mathrm{U}_{2 \vec{\alpha}}$ with $\alpha_{1}=\cdots=\alpha_{l}=\infty$ and $\alpha_{l+1}=\cdots=\alpha_{\mathrm{m}}=0$. Let $Q_{s}=2^{s+3} \sqrt{n} Q \backslash 2^{s+2} \sqrt{n} Q$ for $s=1,2, \cdots$. For $x, y \in Q$ and any $y_{j} \in Q_{s}$ with $j \neq l+1, \cdots, m$, one has $\left|y-y_{j}\right| \geqslant 2^{s} \sqrt{n} l(Q)$, then $\left(\int_{0}^{\infty}\left|K_{t}(y, \vec{y})-K_{t}(x, \vec{y})\right|^{2} \frac{d t}{t}\right)^{1 / 2} \leqslant \frac{C \bar{w}\left(2^{-s}\right)}{\left|2^{s} \sqrt{n}\right|^{m}}$. We have the following estimates

$$
\left|T\left(f_{1 k}^{\infty}, \cdots, f_{l k}^{\infty}, f_{(l+1) k}^{0} \cdots f_{(m) k}^{0}\right)(y)-T\left(f_{1 k}^{\infty}, \cdots, f_{l k}^{\infty}, f_{(l+1) k}^{0} \cdots f_{(m) k}^{0}\right)(x)\right|_{q}
$$




$$
\begin{aligned}
& \leqslant C \mid\left(\int_{0}^{\infty}\left|\int_{\left(\mathbb{R}^{n}\right)^{m}} K_{t}\left(y, y_{1}, \ldots, y_{m}\right) \prod_{j=1}^{l} f_{j k}^{\infty}\left(y_{j}\right) \prod_{j=l+1}^{m} f_{j k}^{0}\left(y_{j}\right) d \vec{y}\right|^{2} \frac{d t}{t}\right)^{\frac{1}{2}} \\
& -\left.\left(\int_{0}^{\infty}\left|\int_{\left(\mathbb{R}^{n}\right)^{m}} K_{t}\left(x, y_{1}, \ldots, y_{m}\right) \prod_{j=1}^{l} f_{j k}^{\infty}\left(y_{j}\right) \prod_{j=l+1}^{m} f_{j k}^{0}\left(y_{j}\right) d \vec{y}\right|^{2} \frac{d t}{t}\right)^{\frac{1}{2}}\right|_{q} \\
& \leqslant C\left(\sum_{k=1}^{\infty}\left|\int_{\left(\mathbb{R}^{n} \backslash 8 \sqrt{n} Q\right)^{m}}\left(\int_{0}^{\infty}\left|K_{t}(y, \vec{y})-K_{t}(x, \vec{y})\right|^{2} \frac{d t}{t}\right)^{1 / 2} \prod_{j=1}^{l}\right| f_{j k}^{\infty}\left(y_{j}\right)\left|\prod_{j=l+1}^{m}\right| f_{j k}^{0}\left(y_{j}\right)|d \vec{y}|^{q}\right)^{1 / q} \\
& \leqslant C \prod_{j=l+1}^{m} \int_{8 \sqrt{n} Q}\left|f_{j}\left(y_{j}\right)\right|_{q_{j}} d y_{j} \int_{\left(\mathbb{R}^{n} \backslash 8 \sqrt{n} Q\right)^{l}} \frac{\bar{\omega}\left(\frac{|y-x|}{\sum_{j=1}^{m}\left|y-y_{j}\right|}\right)}{\left(\sum_{j=1}^{m}\left|y-y_{j}\right|\right)^{m n}} \prod_{j=1}^{l}\left|f_{j}\left(y_{j}\right)\right|_{q_{j}} d y_{1} \cdots d y_{l} \\
& \leqslant C \sum_{s=1}^{\infty} \bar{\omega}\left(2^{-s}\right) \prod_{j=l+1}^{m} \int_{2^{s+3} \sqrt{n} Q}\left|f_{j}\left(y_{j}\right)\right|_{q_{j}} d y_{j} \frac{1}{\mid 2^{(s+3)} \sqrt{n} Q^{m}} \int_{\left(2^{s+3} \sqrt{n}\right)^{)^{l}}} \prod_{j=1}^{l}\left|f_{j}\left(y_{j}\right)\right|_{q_{j}} d y_{j} \\
& \leqslant C|\bar{\omega}|_{L \log L^{l}-\text { Dini }} \prod_{j=1}^{m} \frac{1}{2^{(s+3) n|\sqrt{n} Q|}} \int_{2^{s+3} \sqrt{n} Q}\left|f_{j}\left(y_{j}\right)\right|_{q_{j}} d y_{j} \\
& \leqslant C|\bar{\omega}|_{L \log L^{l}-\operatorname{Dini}} \mathcal{M}\left(|\vec{f}|_{q}\right)(x) .
\end{aligned}
$$

Since $0<\delta<1 / 2$, by Hölder's inequality, we obtain that

$$
\begin{aligned}
& \left(\frac{1}{|Q|} \int_{Q}\left|T\left(f_{1 k}^{\infty}, \cdots, f_{l k}^{\infty}, f_{(l+1) k}^{0} \cdots f_{(m) k}^{0}\right)(y)-T\left(f_{1 k}^{\infty}, \cdots, f_{l k}^{\infty}, f_{(l+1) k}^{0} \cdots f_{(m) k}^{0}\right)(x)\right|_{q}^{\delta} d y\right)^{\frac{1}{\delta}} \\
& \quad \leqslant C \frac{1}{|Q|} \int_{Q}\left|T\left(f_{1 k}^{\infty}, \cdots, f_{l k}^{\infty}, f_{(l+1) k}^{0} \cdots f_{(m) k}^{0}\right)(y)-T\left(f_{1 k}^{\infty}, \cdots, f_{l k}^{\infty}, f_{(l+1) k}^{0} \cdots f_{(m) k}^{0}\right)(x)\right|_{q} d y \\
& \quad \leqslant C|\bar{\omega}|_{\text {Dini-L } \log L} \operatorname{lN}\left(|\vec{f}|_{q}\right)(x) .
\end{aligned}
$$

Hence the proof of Lemma 2.5 is complete.

For positive integers $m$ and $j$ with $1 \leqslant j \leqslant m$, we denote by $\mathcal{C}_{j}^{m}$ the family of all finite subsets $\sigma=\{\sigma(1), \cdots, \sigma(j)\}$ of $\{1, \cdots, m\}$ of $j$ different elements.

Lemma 2.6. Let $0<\delta<\varepsilon<1 / \mathrm{m}$. If $\bar{\omega}$ satisfies the condition (1.2), then

$$
\begin{aligned}
M_{\delta}^{\sharp}\left(T_{\Pi \vec{b}, q} \vec{f}\right)(x) \leqslant & C \prod_{j=1}^{l}\left\|b_{j}\right\|_{B M O}\left(\mathcal{M}_{L(\log L)}^{l}\left(|\vec{f}|_{q}\right)(x)+M_{\mathcal{\varepsilon}}\left(T_{q} \vec{f}\right)(x)\right) \\
& +C \sum_{j=1}^{l-1} \sum_{\sigma \in \mathcal{C}_{j}^{l}} \prod_{i \in \sigma}\left\|b_{i}\right\|_{B M O} M_{\mathcal{E}}\left(T_{\Pi b_{\sigma^{\prime}}, q} \vec{f}\right)(x)
\end{aligned}
$$

holds for any smooth vector function $\left\{\overrightarrow{\mathrm{f}}_{\mathrm{k}}\right\}_{\mathrm{k}=1}^{\infty}$ and for any $\mathrm{x} \in \mathbb{R}^{\mathrm{n}}$, where $\sigma^{\prime}=\{1, \cdots, l\} \backslash \sigma$.

Proof. For any cube Q centered at $x$, we can obtain that

$$
\begin{aligned}
\left(\left.\frac{1}{|\mathrm{Q}|} \int_{\mathrm{Q}}|| \mathrm{T}_{\Pi \overrightarrow{\mathrm{b}}, \mathrm{q}}(\overrightarrow{\mathrm{f}})(z)\right|^{\delta}-|\mathrm{C}|^{\delta} \mid \mathrm{d} z\right)^{1 / \delta} & \leqslant \mathrm{C}\left(\frac{1}{|\mathrm{Q}|} \int_{\mathrm{Q}}\left|\mathrm{T}_{\Pi \overrightarrow{\mathrm{b}}}(\overrightarrow{\mathrm{f}})(z)-\mathrm{c}\right|_{\mathrm{q}}^{\delta} \mathrm{d} z\right)^{1 / \delta} \\
& \leqslant \mathrm{C}\left(\frac{1}{|\mathrm{Q}|} \int_{\mathrm{Q}}\left|\left(\mathrm{b}_{1}(z)-\lambda_{1}\right) \cdots\left(\mathrm{b}_{l}(z)-\lambda_{\mathrm{l}}\right) \mathrm{T}(\overrightarrow{\mathrm{f}})(z)\right|_{\mathrm{q}}^{\delta} \mathrm{d} z\right)^{1 / \delta}
\end{aligned}
$$




$$
\begin{aligned}
& +C \sum_{i=1}^{l-1} \sum_{\sigma \in \mathcal{C}_{i}^{l}}\left(\frac{1}{|Q|} \int_{Q}\left(\prod_{j \in \sigma}\left|b_{j}(z)-\lambda_{j}\right| T_{\Pi b_{\sigma^{\prime}}, q} \vec{f}(z)\right)^{\delta} d z\right)^{1 / \delta} \\
& +C\left(\frac{1}{|Q|} \int_{Q}\left|T\left(\left(b_{1}\left(\cdot \cdot_{1}\right)-\lambda_{1}\right) \cdots\left(b_{l}(\cdot l)-\lambda_{l}\right) \vec{f}\right)(z)-c\right|_{q}^{\delta} d z\right)^{1 / \delta} \\
& =I+I I+I I I,
\end{aligned}
$$

where $C=|c|_{q}$ and $\lambda_{j}=\frac{1}{|8 \sqrt{n} Q|} \int_{8 \sqrt{n} Q} b_{j}(z) d z$ for $j=1, \cdots, l$.

Now we give the estimate for the above terms.

We can choose $1<p_{1}, \cdots$, $p_{l}<\infty$ with $\frac{1}{p_{1}}+\cdots+\frac{1}{p_{l}}+\frac{1}{\varepsilon}=\frac{1}{\delta}$. Since $0<\delta<\varepsilon<1 / \mathrm{m}$, Hölder's inequality gives

$$
I \leqslant C \prod_{j=1}^{l}\left\|b_{j}\right\|_{\text {BMO }} M_{\varepsilon}\left(T_{q} \vec{f}\right)(x) \text {. }
$$

Similarly, we have

$$
\mathrm{II} \leqslant \mathrm{C} \sum_{i=1}^{\mathrm{l}-1} \sum_{\sigma \in \mathcal{C}_{i}^{l}} \prod_{j \in \sigma}\left\|b_{j}\right\|_{\text {BMO }} M_{\mathcal{E}}\left(\mathrm{T}_{\Pi \mathrm{b}_{\sigma^{\prime}}, \mathrm{q}} \overrightarrow{\mathrm{f}}\right)(\mathrm{x}) .
$$

Next, we estimate III. Let $\overrightarrow{f^{\alpha}}=f_{1}^{\alpha_{1}} \cdots f_{m}^{\alpha_{m}}$ and $\vec{f}_{j}=\vec{f}_{j}^{0}+\sum_{\vec{\alpha}, \vec{\alpha} \neq \overrightarrow{0}} \overrightarrow{f^{\alpha}}$, where $\vec{f}_{j}^{0}=\vec{f}_{j} \chi_{8 \sqrt{n} Q}$ and $\vec{\alpha}=$ $\left(\alpha_{1}, \ldots, \alpha_{m}\right), \alpha_{i}=0$ or $\infty$. Then, we have

$$
\begin{aligned}
\left|\mathrm{T}\left(\prod_{i=1}^{l}\left(b_{i}\left(\cdot_{i}\right)-\lambda_{i}\right) \vec{f}\right)(z)-c\right|_{q} \leqslant & T_{q}\left(\prod_{i=1}^{l}\left(b_{i}\left(\cdot \cdot_{i}\right)-\lambda_{i}\right) \vec{f}^{0}\right)(z) \\
& +C \sum_{\vec{\alpha}, \vec{\alpha} \neq \overrightarrow{0}}\left|\left(T\left(\prod_{i=1}^{l}\left(b_{i}\left(\cdot \cdot_{i}\right)-\lambda_{i}\right) \vec{f}^{\alpha}\right)\right)(z)-\left(T\left(\prod_{i=1}^{l}\left(b_{i}(\cdot \cdot i)-\lambda_{i}\right) \vec{f}^{\alpha}\right)\right)(x)\right|_{q},
\end{aligned}
$$

where $c=\sum_{\vec{\alpha}, \vec{\alpha} \neq \overrightarrow{0}}\left(T\left(\prod_{i=1}^{l}\left(b_{i}(\cdot i)-\lambda_{i}\right) f_{1}^{\alpha_{1}} \cdots f_{m}^{\alpha_{m}}\right)\right)(x)$.

By using Kolmogorov's inequality and Theorem 2.4, we obtain that

$$
\begin{aligned}
& \left(\frac{1}{|Q|} \int_{Q}\left|T_{q}\left(\prod_{i=1}^{l}\left(b_{i}(\cdot i)-\lambda_{i}\right) \vec{f}^{0}\right)(z)\right|^{\delta} d z\right)^{1 / \delta} \leqslant C\left\|T_{q}\left(\prod_{i=1}^{l}\left(b_{i}(\cdot \cdot i)-\lambda_{i}\right) \vec{f}^{0}\right)\right\|_{L^{1 / m, \infty}\left(Q, \frac{d}{\mid Q}\right)} \\
& \leqslant C \prod_{j=1}^{l} \frac{1}{|Q|} \int_{Q}\left|b_{j}(z)-\lambda_{j} \| f_{j}(z)\right|_{q_{j}} d z \prod_{j=l+1}^{m} \frac{1}{|Q|} \int_{Q}\left|f_{j}(z)\right|_{q_{j}} d z \\
& \leqslant C \prod_{j=1}^{l}\left\|b_{j}\right\|_{\text {BMO }} \mathcal{M}_{L(\log L)}^{l}\left(|\vec{f}|_{q}\right)(x) \text {. }
\end{aligned}
$$

Now, the arguments in Lemma 2.5 can be applied. If $\alpha_{1}=\cdots=\alpha_{l}=\infty$ and $\alpha_{l+1}=\cdots=\alpha_{m}=0$, by the Minkowski inequality and the smoothness condition (1.1), we have

$$
\begin{aligned}
& \left|T\left(\prod_{i=1}^{l}\left(b_{i}(\cdot i)-\lambda_{i}\right) f_{1 k}^{\infty}, \cdots, f_{l k}^{\infty}, f_{(l+1) k}^{0}, \cdots, f_{m k}^{0}\right)(z)-T\left(\prod_{i=1}^{l}\left(b_{i}(\cdot i)-\lambda_{i}\right) f_{1 k}^{\infty}, \cdots, f_{l k}^{\infty}, f_{(l+1) k}^{0}, \cdots, f_{m k}^{0}\right)(x)\right|_{q} \\
& \quad \leqslant C\left|\left(\int_{0}^{\infty}\left|\int_{\left(\mathbb{R}^{n}\right)^{m}}\right| K_{t}(z, \vec{y})-K_{t}(x, \vec{y})|| \prod_{i=1}^{l}\left(b_{i}\left(y_{i}\right)-\lambda_{i}\right)\left|\prod_{j=1}^{l}\right| f_{j k}^{\infty}\left(y_{j}\right)\left|\prod_{j=l+1}^{m}\right| f_{j k}^{0}\left(y_{j}\right)|d \vec{y}|^{2} \frac{d t}{t}\right)^{\frac{1}{2}}\right|_{q}
\end{aligned}
$$




$$
\begin{aligned}
& \left.\leqslant C\left|\int_{\left(\mathbb{R}^{n}\right)^{m}}\left(\int_{0}^{\infty}\left|K_{t}(z, \vec{y})-K_{t}(x, \vec{y})\right|^{2} \frac{d t}{t}\right)^{1 / 2}\right| \prod_{i=1}^{l}\left(b_{i}\left(y_{i}\right)-\lambda_{i}\right)\left|\prod_{j=1}^{l}\right| f_{j k}^{\infty}\left(y_{j}\right)\left|\prod_{j=l+1}^{m}\right| f_{j k}^{0}\left(y_{j}\right)|d \vec{y}|^{2} \frac{d t}{t}\right)\left.^{\frac{1}{2}}\right|_{q} \\
& \leqslant C \sum_{k=1}^{\infty} \int_{\left(Q_{k}\right)^{l}} \bar{\omega}\left(2^{-k}\right) \frac{\left|\prod_{i=1}^{l}\left(b_{i}\left(y_{i}\right)-\lambda_{i}\right)\right|\left|f_{1}\left(y_{1}\right)\right|_{q_{1}} \cdots\left|f_{l}\left(y_{l}\right)\right|_{q_{l}} d y_{1} \cdots d y_{l}}{\left(\left|z-y_{1}\right|+\cdots+\left|z-y_{m}\right|\right)^{m n}} \prod_{j=l+1}^{m} \int_{8 Q}\left|f_{j}\left(y_{j}\right)\right|_{q_{j}} d y_{j} \\
& \leqslant C \sum_{k=1}^{\infty} \frac{\bar{\omega}\left(2^{-k}\right)}{\left(2^{k}|\sqrt{n} Q|^{\frac{1}{n}}\right)^{m n}} \prod_{j=1}^{l} \int_{2^{k+3} \sqrt{n} Q}\left|b_{j}\left(y_{j}\right)-\lambda_{j}\right|\left|f_{j}\left(y_{j}\right)\right|_{q_{j}} d y_{j} \prod_{j=l+1}^{m} \int_{2^{k+3} \sqrt{n} Q}\left|f_{j}\left(y_{j}\right)\right|_{q_{j}} d y_{j} \\
& \leqslant C \sum_{k=1}^{\infty} \bar{\omega}\left(2^{-k}\right) k^{l} \prod_{j=1}^{l}\left\|b_{j}\right\|_{B M O}\left\|\left|f_{j}\right|_{q_{j}}\right\|_{L(\log L), 2^{k+3} \sqrt{n} Q} \prod_{j=l+1}^{m} \frac{1}{2^{(k+3) n}|\sqrt{n} Q|} \int_{2^{k+3} \sqrt{n} Q}\left|f_{j}\right|_{q_{j}} d y_{j} \\
& \leqslant C|\bar{\omega}|_{L \log L^{l}-\text { Dini }} \prod_{j=1}^{l}\left\|b_{j}\right\|_{B M O} \mathcal{M}_{L(\log L)}^{l}\left(|\vec{f}|_{q}\right)(x),
\end{aligned}
$$

where in the last inequality we used the fact that $\bar{\omega}$ satisfies the condition (1.2).

For the other cases, the estimates are similar. Then we proved Lemma 2.6.

By modifying the proof of Theorem 3.1 in [10], we get the following.

Lemma 2.7. Let $0<\mathrm{p}<\infty, 1 / \mathrm{m}<\mathrm{q}<\infty$, and $\frac{1}{\mathrm{q}_{1}}+\cdots+\frac{1}{\mathrm{q}_{\mathrm{m}}}=\frac{1}{\mathrm{q}}$ with $1<\mathrm{q}_{1}, \cdots, \mathrm{q}_{\mathrm{m}}<\infty$. Suppose that $\overrightarrow{\mathrm{b}} \in(\mathrm{BMO})^{\mathrm{l}}, w \in \mathrm{A}_{\infty}$ and $\overline{\mathrm{w}}$ satisfies the condition (1.2), then

$$
\int_{\mathbb{R}^{n}}\left|\mathrm{~T}_{\Pi \overrightarrow{\mathrm{b}}, \mathrm{q}} \overrightarrow{\mathrm{f}}\right|^{\mathrm{p}} \mathcal{w}(\mathrm{x}) \mathrm{d} x \leqslant \mathrm{C} \prod_{j=1}^{\mathrm{l}}\left\|\mathrm{b}_{j}\right\|_{\mathrm{BMO}}^{\mathrm{p}} \int_{\mathbb{R}^{n}}\left(\mathcal{M}_{\mathrm{L}(\log \mathrm{L})}^{\mathrm{l}}\left(|\overrightarrow{\mathrm{f}}|_{\mathrm{q}}\right)(\mathrm{x})\right)^{\mathrm{p}} \mathcal{w}(\mathrm{x}) \mathrm{d} x,
$$

and

$$
\begin{aligned}
& \sup _{t \rightarrow 0} \frac{1}{\Phi^{(m)}(1 / t)} \omega\left(\left\{y \in \mathbb{R}^{n}:\left|T_{\Pi \vec{b}, q} \vec{f}(y)\right|>t^{m}\right\}\right) \\
& \leqslant C \sup _{t>0} \frac{1}{\Phi^{(m)}(1 / t)} \omega\left(\left\{y \in \mathbb{R}^{n}: \mathcal{M}_{L(\log L)}^{l}\left(|\vec{f}|_{q}\right)(y)>t^{m}\right\}\right),
\end{aligned}
$$

hold for any smooth function $\overrightarrow{\mathrm{f}}$ with compact support.

The following multiple weighted estimates for $\mathcal{M}_{\mathrm{L}(\log \mathrm{L})}^{\mathrm{l}}$ can be found in [10].

Lemma 2.8 ([10]). Let $1 / \mathrm{m}<\mathrm{q}<\infty$ and $1<\mathrm{q}_{1}, \cdots, \mathrm{q}_{\mathrm{m}}<\infty$ with $\frac{1}{\mathrm{q}}=\frac{1}{\mathrm{q}_{1}}+\cdots+\frac{1}{\mathrm{q}_{\mathrm{m}}}$.

(i) Let $1 / \mathrm{m}<\mathrm{p}<\infty$ and $1<\mathrm{p}_{1}, \cdots, \mathrm{p}_{\mathrm{m}}<\infty$ with $\frac{1}{\mathrm{p}}=\frac{1}{\mathrm{p}_{1}}+\cdots+\frac{1}{\mathrm{p}_{\mathrm{m}}}$, and $\vec{\omega}$ satisfies the $A_{\vec{p}}$ condition.

Then

$$
\left(\int_{\mathbb{R}^{n}}\left|\mathcal{M}_{\mathrm{L}(\log \mathrm{L})}^{\mathrm{l}}\left(|\overrightarrow{\mathrm{f}}|_{\mathrm{q}}\right)(\mathrm{x})\right|^{\mathrm{p}} v_{\overrightarrow{\boldsymbol{\omega}}}(\mathrm{x}) \mathrm{d} x\right)^{1 / \mathrm{p}} \leqslant \mathrm{C}\left(\left.\left.\int_{\mathbb{R}^{n}}|| \mathrm{f}_{j}\right|_{q_{j}}(x)\right|^{p_{j}} \omega_{j}(x) \mathrm{d} x\right)^{1 / p_{j}} .
$$

(ii) Let $\vec{\omega} \in A_{(1, \cdots, 1)}$. Then

$$
v_{\vec{w}}\left(\left\{x \in \mathbb{R}^{n}: \mathcal{M}_{L(\log L)}^{l}\left(|\vec{f}|_{q}\right)(x)>t^{m}\right\}\right) \leqslant C\left(\prod_{j=1}^{m} \int_{\mathbb{R}^{n}} \Phi^{(m)}\left(\frac{\left|f_{j}\right|_{q_{j}}\left(y_{j}\right)}{t}\right) \omega_{j}\left(y_{j}\right) d y_{j}\right)^{1 / m}
$$

where $\Phi(\mathrm{t})=\mathrm{t}\left(1+\log ^{+} \mathrm{t}\right)$ and $\Phi^{(\mathrm{m})}=\overbrace{\Phi \circ \cdots \circ \Phi}^{\mathrm{m}}$.

Proof of Theorem 1.3. Combining (2.1) and Lemma 2.8, we get Theorem 1.3.

Proof of Theorem 1.4. Using the properties of function $\Phi$, Lemma 2.7, and Lemma 2.8, we can obtain Theorem 1.4. Since the arguments are almost the same as the proof of Theorem 3.16 in [8], we omit the details here. 


\section{Acknowledgment}

The work was supported by the National Natural Science Foundation of China (No.11401175 and No.11501169).

\section{References}

[1] K. F. Andersen, R. T. John, Weighted inequalities for vector-valued maximal functions and singular integrals, Studia Math., 69 (1980), 19-31. 2

[2] R. R. Coifman, D. G. Deng, Y. Meyer, Domaine de la racine carrée de certains opérateurs différentiels accrétifs, (French) [[Domain of the square root of certain accretive differential operators]], Ann. Inst. Fourier (Grenoble), 33 (1983), 123-134. 1

[3] D. Cruz-Uribe, J. M. Martell, C. Pérez, Extrapolation from $A_{\infty}$ weights and applications, J. Funct. Anal., 213 (2004), 412-439. 2

[4] G. David, J. L. Journé, Une caractérisation des opérateurs intégraux singuliers bornés sur $\mathrm{L}^{2}\left(\mathbf{R}^{\mathrm{n}}\right)$, (French) [[A characterization of singular integral operators bounded on $\left.\mathrm{L}^{2}\left(\mathbf{R}^{\mathrm{n}}\right)\right]$ ], C. R. Acad. Sci. Paris Sér. I Math., 296 (1983), 761-764. 1

[5] E. B. Fabes, D. S. Jerison, C. E. Kenig, Multilinear Littlewood-Paley estimates with applications to partial differential equations, Proc. Nat. Acad. Sci. U.S.A., 79 (1982), 5746-5750. 1

[6] E. B. Fabes, D. S. Jerison, C. E. Kenig, Multilinear square functions and partial differential equations, Amer. J. Math., 107 (1985), 1325-1368.

[7] J. Hart, Bilinear square functions and vector-valued Caldern-Zygmund operators, J. Fourier Anal. Appl., 18 (2012), 1291-1313. 1

[8] A. K. Lerner, S. Ombrosi, C. Pérez, R. H.Torres, R. Trujillo-González, New maximal functions and multiple weights for the multilinear Calderón-Zygmund theory, Adv. Math., 220 (2009), 1222-1264. 2, 2.1, 2

[9] S. Sato, K. Yabuta, Multilinearized Littlewood-Paley operators, Sci. Math. Jpn., 55 (2002), 447-453. 1

[10] Z.-Y. Si, Q.-Y. Xue, Weighted estimates for commutators of vector-valued maximal multilinear operators, Nonlinear Anal., 96 (2014), 96-108. 2, 2, 2.8

[11] Z.-Y. Si, Q.-Y. Xue, Multilinear square functions with kernels of Dini's type, J. Funct. Spaces, 2016 (2016), 11 pages. 1.2, 2.3

[12] Q.-Y. Xue, J.-Q. Yan, On multilinear square function and its applications to multilinear Littlewood-Paley operators with non-convolution type kernels, J. Math. Anal. Appl., 422 (2015), 1342-1362. 1, 1.1

[13] K. Yabuta, A multilinearization of Littlewood-Paley's g-function and Carleson measures, Tôhoku Math. J., 34 (1982), 251-275. 1 